oder Polio kann geimpft werden. Die Notwendigkeit von Impfungen gegen Cholera, FSME, Pertussis, Pneumokokken, Hepatitis A und B sollte sehr streng abgewogen werden.

In Gelbfieber-Regionen sollten Schwangere möglichst nicht reisen, obwohl Schädigungen durch die Lebendvakzine in der Schwangerschaft bisher nicht beschrieben wurden. Sollte eine Reise aber unvermeidbar sein, kann die Impfung durchgeführt werden, aber möglichst nicht im 1. Trimenon.

Wird die Impfung aber als reine Vorschrift bei der Einreise verlangt ohne konkretes Risiko, kann man der Schwangeren ein Impfbefreiungszeugnis aus medizinischer Indikation auf die Reise mitgeben. Es ist jedoch nicht sicher, ob alle Länder dies wirklich akzeptieren. (Vgl. Übersicht auf S. 70)

\section{Mutterpass mitführen}

Die IATA hat Rahmenempfehlungen herausgegeben, wann Schwangere fliegen dürfen. Danach sollte die Frau etwa ab der 29. Woche ein Unbedenklichkeitsattest ihres Frauenarztes beim Check In vorlegen. Bis zur 32. Woche dürfen Frauen mit Mehrlingsschwangerschaft, bis zur 36. Woche mit Einlingsgravidität fliegen. Ab dem 7. Tag post partum sind Mutter und Kind wieder flugtauglich. Da die Regeln von den Fluggesellschaften unterschiedlich gehandhabt werden, sollte sich die Schwangere unbedingt vorab bei ihrer Airline informieren. Außerdem sollte sie ihren Mutterpass im Handgepäck mitführen für den Fall, dass er beim Check-in verlangt wird.

\section{MARTIN BISCHOFF :}

- Symposium „Aktuelle Reisemedizin: Besondere Reisesituationen", DGIM 2012, Wiesbaden, 14.-17. April 2012

\title{
Nur wer brennt, kann andere entflammen
}

Der Vorstand der Vereinigung der Deutschen Medizinischen Fach- und Standespresse e.V. (VMWJ) verleiht seit 1974 jährlich den Walter-Trummert-Preis an verdiente Medizinjournalisten. In diesem Jahr geht der Preis an Dr. med. Dirk Einecke, Chefredakteur der MMW-Fortschritte der Medizin, Herausgeber und Mitglied der Geschäftsleitung in der Verlagsgruppe SpringerMedizin. Mit diesem Preis würdigen die Medizinjournalisten die besonderen Verdienste von Dirk Einecke um die medizinische Publizistik.

Bereits während des Medizinstudiums in Heidelberg konnte Dirk Einecke erste journalistische Erfahrungen im Dienste der "Medical Tribune" in Wiesbaden gewinnen. Nach Approbation und Promotion begann er seine steil verlaufene medizinjournalistische Karriere als Redakteur bei dieser Zeitschrift. Nur drei Jahre später erkannte Jochen Aumiller, damaliger Chefredakteur der MMW, Eineckes Talent und holte ihn als seinen Stellvertreter nach München. Besonderes Aufsehen in der Fachwelt erregte die kaum für möglich gehaltene, aber letztlich erfolgreiche Fusion der MMW mit der bei Urban \& Vogel erschienenen Zeitschrift "Fortschritte der Medizin" unter der Stabführung von Dirk Einecke. Seit 1999 leitete er die so entstandene "MMW-Fortschritte der Medizin" als verantwortlicher Chefre-

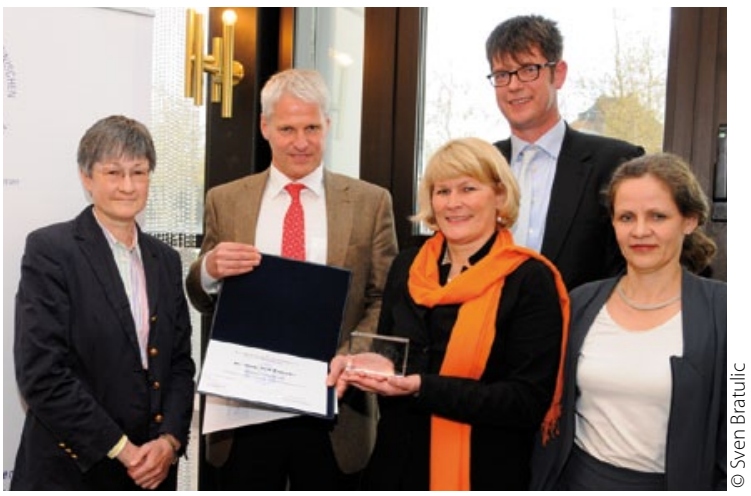

Bei der Preisverleihung: die VMWJ-Vorstandsmitglieder Dr. Susanne Heinzl, Dr. Julia Rautenstrauch und Dr. Wiebke Kathmann (vorne von li.) sowie Dr. Dirk Einecke (li.) und Harm van Maanen (re.), Executive Vice President von SpringerMedizin, der die Laudatio hielt.

dakteur. Dirk Einecke steuert seitdem nicht nur das traditionsreiche Flaggschiff der ärztlichen Fortbildung sicher durch schweres Wetter, sondern legte auch mehrere kleine Beiboote auf Kiel wie "Cardiovasc", „GastroNews", „PneumoNews" und "InfoDiabetologie". Mit großem verlegerischen Geschick gelang ihm die Neupositionierung zahlreicher etablierter Fachzeitschriften innerhalb der Verlagsgruppe SpringerMedizin. Von der journalistischen Brillanz und der ärztlichen Fachkompetenz von Dirk Einecke können sich die Leser der MMW-Fort- schritte der Medizin seit vielen Jahren in jedem Heft überzeugen. Seine Begeisterungsfähigkeit für den Medizinjournalismus hat zahlreiche begabte Redakteure angezogen, die ihm auf dem Weg zu höchster journalistischer Qualität folgen.

Dirk Einecke ist nach Jochen Aumiller bereits der zweite Chefredakteur der MMW, der den im Andenken an den früh verstorbenen Medizinjournalisten Walter Trummert gestifteten Preis erhält. Das Renommee dieses Preises wiegt besonders schwer, da er von der Vereinigung der Deutschen Medizinischen Fach- und Standespresse gestiftet und verliehen wird, die mit über 700 Mitgliedern die größte medizinjournalistische Fachorganisation in Deutschland bildet. Schriftleitung, Verlag und Redaktion der "MMW-Fortschritte der Medizin" preisen sich glücklich, einen Mann wie Dirk Einecke an der Spitze zu haben und gratulieren herzlich.

PROF. DR. H. S. FÜESSL = 